\title{
BAG3 regulates epithelial-mesenchymal transition and angiogenesis in human hepatocellular carcinoma
}

\author{
Heng Xiao ${ }^{1,2}$, Shaobing Cheng ${ }^{1,2}$, Rongliang Tong ${ }^{1,2}$, Zheng Lv ${ }^{1}$, Chaofeng Ding ${ }^{1}$, Chengli Du', \\ Haiyang $\mathrm{Xie}^{2}$, Lin $\mathrm{Zhou}^{2}$, Jian $\mathrm{Wu}^{2}$ and Shusen Zheng ${ }^{1,2}$
}

Bcl2-associated athanogene 3 (BAG3) protein is a co-chaperone of heat-shock protein ( $\mathrm{Hsp}$ ) 70 and may regulate major physiological and pathophysiological processes. However, few reports have examined the role of BAG3 in human hepatocellular carcinoma (HCC). In this study, we show that BAG3 regulates epithelial-mesenchymal transition (EMT) and angiogenesis in HCC. BAG3 was overexpressed in HCC tissues and cell lines. BAG3 knockdown resulted in reduction in migration and invasion of HCC cells, which was linked to reversion of EMT by increasing E-cadherin expression and decreasing $\mathrm{N}$-cadherin, vimentin and slug expression, as well as suppressing matrix metalloproteinase 2 (MMP-2) expression. In a xenograft tumorigenicity model, BAG3 knockdown effectively inhibited tumor growth and metastasis through reduction in CD34 and VEGF expression and reversal of the EMT pathway. In conclusion, BAG3 is associated with the invasiveness and angiogenesis in HCC, and the BAG3 gene may be a novel therapeutic approach against HCC.

Laboratory Investigation (2014) 94, 252-261; doi:10.1038/labinvest.2013.151; published online 23 December 2013

KEYWORDS: Bcl2-associated athanogene 3; human hepatocellular carcinoma; epithelial-mesenchymal transition; angiogenesis

$\mathrm{Bcl} 2$-associated athanogene 3 (BAG3) protein is one of six BAG family proteins, all of which contain a conserved domain that interacts with the heat-shock protein (Hsp) $70 .^{1-3}$ BAG3 is expressed in many primary tumors or tumor cell lines, such as leukemia, thyroid, neuroblastoma, prostate carcinomas, pancreas, ovarian cancer, and glioblastoma. ${ }^{4-10}$ It has been reported that BAG3 may regulate diverse biological processes, including apoptosis, development, cytoskeleton organization, and autophagy. ${ }^{11}$ Some studies have shown that BAG3 upregulation results in inhibition of drug-induced or serum-deprivation apoptosis, and BAG3 downregulation in glioblastoma results in reduction in tumor growth. ${ }^{5,10}$ Furthermore, it has been reported that BAG3 controls angiogenesis in endothelial cells and tumor cells through ERK phosphorylation, and thereby contributes to tumor growth in vivo. ${ }^{12}$ BAG3 was also reported to interact with matrix metalloproteinase 2 (MMP-2) and to induce epithelial-mesenchymal transition (EMT), thus regulating the process of tumor migration and invasion. ${ }^{9,13}$

However, little is known about the role of BAG3 in hepatocellular carcinoma (HCC). HCC, a highly vascular and invasive tumor, is the third leading cause of cancer death worldwide, and the second in China. ${ }^{14,15}$ Because existing therapies are insufficient for tumor eradication and the high frequency of tumor recurrence, the prognosis for HCC patients remains pessimistic. It is therefore important to determine the molecular mechanisms of angiogenesis and metastasis in HCC, and to establish the identity of new therapeutic targets for that will ultimately improve the prognosis for HCC patients.

In this report, we used HCC cell lines to investigate the expression of BAG3 and to assess the biological function of the BAG3 protein, with a focus on angiogenesis and metastasis. Our results suggest that BAG3 is highly expressed in many HCC cell lines, and that reduction in BAG3 expression suppresses HCC cell migration and invasion in vitro, and growth and angiogenesis in vivo.

\section{MATERIALS AND METHODS Clinical Specimen Collection}

Samples from 65 patients receiving hepatic resection at our hospital (First Affiliated Hospital, Zhejiang University School

\footnotetext{
${ }^{1}$ Division of Hepatobiliary and Pancreatic Surgery, Department of Surgery, First Affiliated Hospital, Zhejiang University School of Medicine, Hangzhou, China and ${ }^{2}$ Key Lab of Combined Multi-Organ Transplantation, Ministry of Public Health, Hangzhou, China

Correspondence: Professor J Wu, Key Lab of Combined Multi-Organ Transplantation, Ministry of Public Health, Hangzhou 310003, China or Dr S Zheng, Division of Hepatobiliary and Pancreatic Surgery, Department of Surgery, First Affiliated Hospital, Zhejiang University School of Medicine, Hangzhou 310003 , China.

E-mail: drwujian@hotmail.com or shusenzheng@zju.edu.cn

Received 15 July 2013; revised 10 November 2013; accepted 11 November 2013
} 
of Medicine, Zhejiang, China) between 2005 and 2010 were collected for this study. Letters of consent were obtained from all patients, and the experimental protocols were approved by the local ethics committee. Patient charts were reviewed to obtain clinical data about age, gender, tumor size, AFP, HBsAg, Portal vein-emboli and metastasis, TNM stage (AJCC), and tumor differentiation.

\section{Cell Culture}

Six human HCC cell lines (HepG2, Hep3B, Huh-7, Bel-7402, SK-Hep-1, SMMC-7721, and MHCC-LM3) and one immortalized liver cell line (L-02) were purchased from Cell Bank of Type Culture Collection of Chinese Academy of Sciences, Shanghai Institute of Cell Biology, Chinese Academy of Sciences and were cultivated as described by the suppliers. HepG2, Hep3B, Huh-7, SK-Hep-1, and MHCCLM3were were cultured in Dulbecco's modified Eagle medium (DMEM; Gibco-Invitrogen, Carlsbad, CA, USA) supplemented with $10 \%$ fetal bovine serum. Bel-7402, SMMC-7721, and L-02 were cultured in 1640 complete medium supplemented with $10 \%$ fetal bovine serum.

\section{Antibodies}

Rabbit monoclonal antibodies recognizing BAG3 (Cat.\#: 2783-1, dilution 1/1000), VEGF-A (Cat.\#: 1909-1, dilution 1/ 1000), MMP-9 (Cat.\#: 1939-1, dilution 1/1000), MMP-2 (Cat.\#: 1948-1, dilution 1/1000), CD34 (Cat.\#: 2150-1, dilution 1/200), HIF-1a (Cat.\#: 2015-1, dilution 1/1000), $\beta$-actin (Cat.\#: 1854-1, dilution 1/1000), and GAPDH (Cat.\#: 5632-1, dilution 1/1000) were obtained from Epitomics, an ABCAM company (Cambridge, MA, USA). EMT-related antibodies (NO.9782S, dilution 1/1000) (E-cadherin, $\mathrm{N}$-cadherin, slug, snail, and vimentin) were obtained from Cell Signaling Technology (Danvers, MA, USA).

\section{Transfections of Lentiviral Vectors with BAG3 siRNA}

To study the function of BAG3, a BAG3 siRNA lentiviral vector (lenti-siRNA/BAG3) was constructed (Shanghai GeneChem Co., Ltd., Shanghai, China). A GFP-lentiviral vector (Scr-siRNA/GFP) was used as a negative control. Double-stranded oligonucleotides encoding human BAG3 siRNA (NM_004281, 5'-TGAACAGAAAGCCATTGAT-3') were inserted into the siRNA expression vector Scr-siRNA/ GFP (Shanghai GeneChem Co., Ltd.). All lentiviral vectors expressed GFP, which allowed us to measure the infection efficiency. Then, $5 \times 10^{5}$ LM3 and SMMC-7721 cells were plated into 24 -well plates and incubated for $24 \mathrm{~h}$. The lentiviruses were transected according to the manufacturer's instructions. For stable cell lines, transfected cells were selected using $3 \mu \mathrm{g} / \mathrm{ml}$ of puromycin.

\section{Total RNA Extraction and RT-PCR}

A TRIzol Total RNA extraction kit (Invitrogen Co.) was used for total RNA extraction; a Rever Tre Ace-a-reverse transcription kit (Invitrogen Co.) was used to synthesize
cDNA; for quantitative real-time RT-PCR analysis, the Roche LightCycler was used with Takara SYBR Premix Extaq system. All procedures were performed following the instructions from the kits. Primers were synthesized by Shanghai Sangon Biological Engineering Technology Services Co., Ltd. The nucleotide sequences of the primers were as follows: GAPDH, $5^{\prime}$-ATGGGGAAGGTGAAGGTCG-3' and $5^{\prime}$-GGGG TCATTGATGGCAACAATA-3'; BAG3, 5'-TGGGAGATCAAG ATCGACCC-3' and $5^{\prime}$-GGGCCATTGGCAGAGGATG-3'; HIF-1a, 5'-ATCCATGTGACCATGAGGAAATG- $3^{\prime}$ and $5^{\prime}$-TC GGCTAGTTAGGGTACACTTC-3'; E-cadherin, 5'-CGAGAG CTACACGTTCACGG- ${ }^{\prime}$ and $5^{\prime}$-GGGTGTCGAGGGAAAAA TAGG-3'; N-cadherin, 5'-TTTGATGGAGGTCTCCTAACA CC-3' and $5^{\prime}$-ACGTTTAACACGTTGGAAATGTG-3'; Vimentin, $5^{\prime}$-GACGCCATCAACACCGAGTT- $3^{\prime}$ and $5^{\prime}$-CTTTGTCGTTG GTTAGCTGGT-3'; Slug, 5'-CGAACTGGACACACATACAGT G-3' and $5^{\prime}$-CTGAGGATCTCTGGTTGTGGT- $3^{\prime}$; VEGF, 5'-AG GGCAGAATCATCACGAAGT- $3^{\prime}$ and $5^{\prime}$-AGGGTCTCGATTGG ATGGCA-3' ${ }^{\prime}$. Each sample was taken in triplicate. GAPDH was used as an internal reference, and the $2-\Delta \Delta \mathrm{CT}$ method was used to analyze PCR results.

\section{Western Blotting}

After incubation, cells were lysed and sonicated. Soluble proteins were collected and stored at $-80^{\circ} \mathrm{C}$ after a centrifugation at $12000 \mathrm{~g}$ for $15 \mathrm{~min}$. Protein concentration was determined by the Bradford assay (Bio-Rad). After denaturation, the proteins were separated by gel electrophoresis using 10\% SDS-PAGE, and then transferred onto PVDF membrane for $2 \mathrm{~h}$, followed by blocking in 5\% skim milk. The membrane was washed once with TBST, then incubated for overnight at $4{ }^{\circ} \mathrm{C}$ with relevant antibodies (1:1000). The membrane was again washed three times with TBST, then incubated with secondary antibody (Goat Anti-Rabbit IgG $1: 2000)$ for $2 \mathrm{~h}$ at room temperature. The membrane was washed third time with TBST and then ECL liquid was added and placed in a darkroom for the reaction to proceed. $\beta$-Actin and GAPDH (1:1000) were used as positive controls.

\section{Cell Proliferation Assay}

Cell proliferation was determined by the cell counting assay- 8 (CCK-8) cell viability assay (Dojindo Laboratories, Kumamoto, Japan). Cell was seeded into 96-well plates at a density of $5 \times 10^{3}$ cells per well. After incubation for $48 \mathrm{~h}$, the supernatant was changed with $100 \mu \mathrm{l}$ medium and $10 \mu \mathrm{l} \mathrm{CCK-}$ 8 was added and cells were incubated for $1 \mathrm{~h}$. The OD values were quantified by Versamax microplate reader at $450 \mathrm{~nm}$.

\section{Cell Migration and Invasion Assays}

For the transwell migration assays, $5 \times 10^{4}$ LM3 cells or $5 \times 10^{4}$ SMMC-7721 cells which had been permanently transfected in serum-free medium were placed on the upper chamber with a non-coated membrane (24-well insert; 8-mm pore size; Millipore, Billerica, MA, USA). For invasion assays, $5 \times 10^{4}$ LM3 cells or $5 \times 10^{5}$ SMMC-7721 cells in serum-free 
a

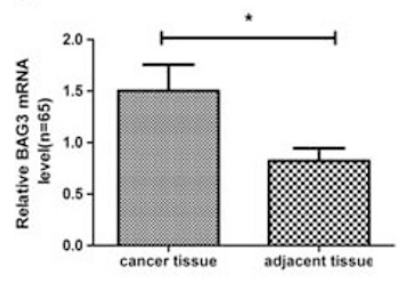

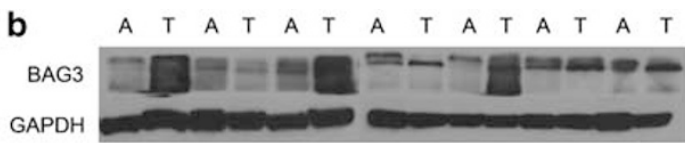

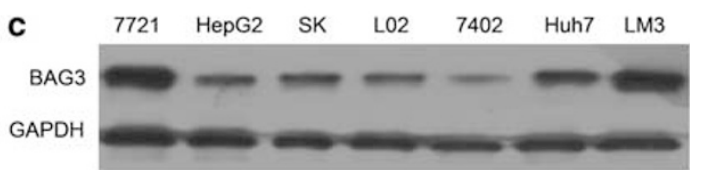

Figure 1 BAG3 is highly expressed in HCC tissues and cell lines. (a) Relative BAG3 mRNA expression was measured by real-time PCR in 65 HCC tissues. (b) BAG3 protein and EMT markers (E-cadherin, $\mathrm{N}$-cadherin, vimentin, and slug) expression was determined by western blotting in 12 HCC tissues. BAG3 protein expression was determined by western blotting in six HCC cell lines. GAPDH and $\beta$-actin were used as controls. ' $C$ ' represents cancer tissue and 'N' represents non-cancerous tissue. Data are expressed as mean \pm s.d. ${ }^{*} P<0.05$.

medium were placed in the upper chamber with a Matrigelcoated membrane. In both assays, culture medium containing $10 \%$ FBS was added in the lower chamber. The cells were incubated for $24 \mathrm{~h}$ (migration assay) and $48 \mathrm{~h}$ (invasion assay). Cells on the lower membrane were fixed with $100 \%$ methanol, stained with $0.2 \%$ crystal violet and counted.

\section{Colony-Forming Assay}

Log-phase cells (600 cells/well) were seeded onto six-well plates with $2 \mathrm{ml}$ of $10 \%$ FBS medium and incubated at $37^{\circ} \mathrm{C}$ in a humidified incubator. Ten to twelve days after plating, the colonies were fixed with $100 \%$ methanol, stained with $0.2 \%$ crystal violet and counted.

\section{Tumor Xenograft Experiments}

All experimental procedures were performed in accordance with the National Institutes of Health guide for the care and use of laboratory animals. In all, $1 \times 10^{7}$ cells were resuspended in $100 \mu \mathrm{l}$ PBS, and injected subcutaneously into the lateral flanks of immunodeficient mice. Tumor volumes were measured weakly, with calculation using the equation: $V\left(\mathrm{~cm}^{3}\right)=$ width $\left(\mathrm{cm}^{2}\right) \times$ length $(\mathrm{cm}) / 2$. After 4 weeks, tumors were harvested for immunostaining after tumor implantation.

\section{Statistical Analysis}

SPSS17.0 software was used for statistical analysis. The experimental data were expressed as mean \pm s.d., and assessed by a two-tailed Student's $T$ test. The chi-square test or Fisher's exact test was used to evaluate any potential association between the BAG3 expression and the clinicopathologic parameters. Statistical significance was accepted if $P<0.05$.

\section{RESULTS}

\section{BAG3 Is Highly Expressed in HCC and HCC Cell Lines}

To analyze the expression of BAG3 in HCC, 65 patients' tumor tissues and tissues adjacent to the cancer were examined by RT-PCR. As seen in Figure 1a, the level of BAG3 mRNA was significantly higher in HCC than in adjacent tissues $(P=0.017)$. To verify this result, we analyzed EMT markers by western blotting, re-blotting the other 12 pairs of samples. Nine samples of the tumor tissue showed overexpression of BAG3 compared with adjacent tissue, and the mesenchymal marker slug showed changes similar to those found for BAG3. However, epithelial markers (such as E-cadherin) and mesenchymal markers (such as N-cadherin and vimentin) did not show such a correlation (Figure 1b). We then analyzed the relationship between BAG3 expression and clinicopathologic features of the tumors. Because seven patients had no detailed clinical data, we were only able to analyze 58 patients' clinical data. Intriguingly, we found that BAG3 expression levels were significantly higher in HCC with portal vein-emboli and metastasis $(P=0.007$; Table 1$)$. Thus, BAG3 expression appears to be a valuable predictive factor for tumor metastasis and HCC aggressiveness. Examination of BAG3 protein expression in six HCC cell lines (SMMC7721, HepG2, SK-hep-1, Bel-7402, Huh7, and MHCC-LM3) and one immortalized liver cell line (L-02) showed that BAG3 was expressed in all of these cell lines. At least five of these HCC lines (SMMC-7721, HepG2, SK-hep-1, Huh7, and MHCC-LM3) showed increased protein expression compared with the immortalized liver cell line (L-02) (Figure 1c). For subsequent experiments, low-invasion and high-invasion HCC cell lines (SMMC-7721 and MHCC-LM3) were selected due to their invasion and BAG3 expression characteristics.

\section{Establishment of BAG3 Knockdown HCC Cell Lines}

To study the mechanism of BAG3 in HCC, we established BAG3 knockdown cell lines. For stable knockdown of BAG3, SMMC-7721 and MHCC-LM3 cells were transfected with a GFP-lentiviral vector (Scr-siRNA/GFP) which was also used as a negative control, and BAG3 siRNA. Stable cell lines were selected using puromycin. In stable cell lines, BAG3 mRNA level was measured by quantitative RT-PCR. Compared with the control group, BAG3 transcripts were significantly reduced in BAG3-siRNA-transfected cells $(P=0.002$, $P=0.004$ ) (Figures $2 \mathrm{a}$ and $\mathrm{b}$ ). Accordingly, the BAG3 protein levels were markedly decreased in BAG3-siRNA-transfected cells as shown by western blotting (Figure 2c). These results demonstrate that BAG3-siRNA transfection leads to the reduction in BAG3 expression. 
Table 1 Relationship between BAG3 expression and clinicopathologic features

\begin{tabular}{|c|c|c|c|}
\hline \multirow[t]{2}{*}{ Variable } & \multicolumn{2}{|c|}{ BAG3 expression } & \multirow[t]{2}{*}{$P$-value ${ }^{a}$} \\
\hline & Low BAG3 & High BAG3 & \\
\hline
\end{tabular}

\section{In general}

Tumor tissue

26

Sex

Male

Female

Age (years)

$\leqq 50$

$>50$

Tumor size $(\mathrm{cm})$

$\leqq 5$

$>5$

$\operatorname{AFP}(\mathrm{ng} / \mathrm{ml})$
$\quad \leqq 400$
$>400$

HBsAg

Positive

25

Portal vein-emboli and metastasis

$$
\text { Yes }
$$

No

5
21

TNM stage (AJCC)
I-II
III-IV

Tumor differentiation

$\begin{array}{llll}\text { I-II } & 10 & 10 & 0.591 \\ \text { III-IV } & 16 & 22 & \end{array}$

${ }^{\text {a }}$ Statistical analyses were performed with chi-square test or Fisher's exact test. ${ }^{*} P<0.05$.

Knockdown of BAG3 Induces Apoptosis of HCC Cell Lines To clarify the effect of BAG3 RNAi on apoptosis in HCC cells, a flow-cytometry method was employed. We found that suppression of BAG3 could induce apoptosis of SMMC-7721 and LM3 cells as quantified by PE/7AAD staining. SMMC-
7721 cells with BAG3 RNAi experienced more apoptosis $(10.24 \pm 0.28)$ than negative control cells did $(5.33 \pm 0.31$, $P=0.007)$, or LM3 cell lines $(27.10 \pm 0.30$ vs $12.79 \pm 0.78$, $P=0.003$ ) (Figure 3).

\section{Knockdown of BAG3 Inhibits Migration and Invasion of HCC Cell Lines}

A transwell assay was used to study whether knockdown of BAG3 would impact migration and invasion of HCC cell lines. The BAG3-siRNA-transfected cells showed significantly decreased motility compared with the controls (Figure 4a). Because tumor cell migration is a key factor which results in cancer invasion, an invasion assay was also performed following BAG3 knockdown. The BAG3-siRNA-transfected cells showed a significant decrease in invasiveness compared with the controls $(P<0.001)$ (Figure 4a). Because the cell invasion assay chamber was incubated for $48 \mathrm{~h}$, cell growth might impact the cell invasion rate. A CCK8 assay was used to determine whether knockdown of BAG3 could affect the proliferation of HCC cell lines. There was no significant proliferation noted at $48 \mathrm{~h}$ (Figure $4 \mathrm{~b}$ ). MMP-mediated extracellular matrix (ECM) degradation is a crucial step in cell invasion. Western blotting was therefore performed to find out whether knockdown of BAG3 could affect MMP expression. The reduction in BAG3 expression led to a decrease in MMP-2 protein expression, but the MMP-9 protein level remained unchanged (Figure 4c).

\section{BAG3 Knockdown Reduces EMT in HCC Cell Lines}

EMT is a crucial step in cellular invasion and metastasis. To investigate whether knockdown of BAG3 expression changes the expression of EMT molecules in HCC cells, we used quantitative RT-PCR and western blotting to analyze EMT markers. BAG3-siRNA-transfected cells exhibited upregulation of mRNA for epithelial marker E-cadherin, and downregulation of mRNA for the mesenchymal markers $\mathrm{N}$-cadherin and slug, compared with the controls $(P<0.001)$ (Figure 5a). Consistent with mRNA expression, western blot analysis and immunofluorescence analysis showed that BAG3 knockdown induced higher E-cadherin protein expression, and lower $\mathrm{N}$-cadherin and slug protein expression (Figures $5 \mathrm{~b}$ and $\mathrm{c}$ ). Although vimentin mRNA showed no significant change in BAG3-siRNA-transfected SMMC-7721 cells and vimentin mRNA expression was increased in BAG3siRNA-transfected MHCC-LM3 cells (Figure 5a), vimentin protein expression was significantly decreased in both of the BAG3-siRNA-transfected cells (Figures $5 \mathrm{~b}$ and c).

BAG3 Knockdown Inhibits HCC Cells Colony Formation In Vitro and Tumorigenicity of Transfected Cancer Cell Lines In Vivo

BAG3-siRNA-transfected cells showed significantly fewer colonies than the control groups (Figure 6a). We further investigated the effect of BAG3 on HCC growth in nude mice. BAG3-siRNA-transfected SMMC-7721 cells and negative 
a

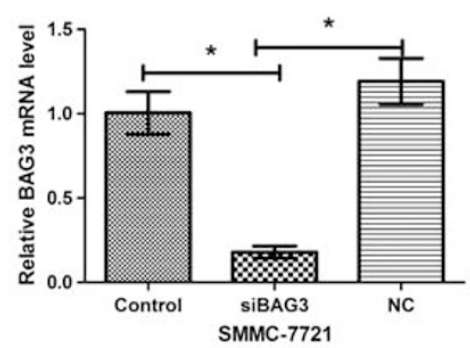

C

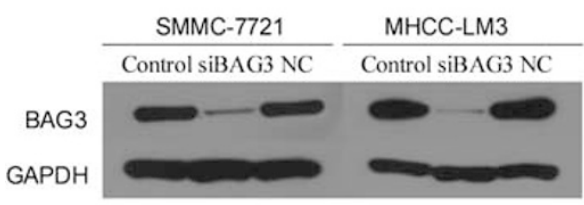

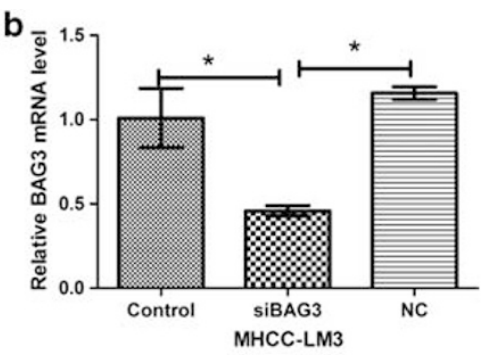

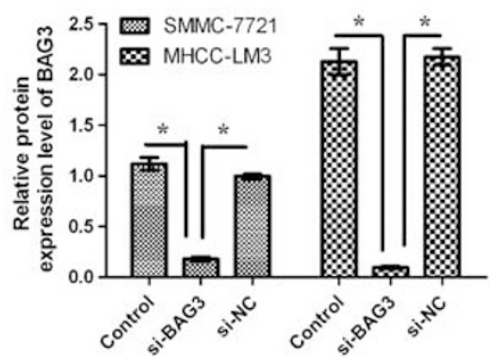

Figure 2 Establishment of BAG3 knockdown HCC cell lines. (a, b) SMMC-7721 and MHCC-LM3 HCC cell lines were transfected with non-targeting siRNA (NC) or BAG3 siRNA, and the expression of BAG3 was measured by real-time PCR after stable cell lines were selected. (c) The protein levels of stable BAG3 knockdown HCC cell lines were measured by western blotting. GAPDH was used as a control. Experiments were performed three times. Data were expressed as mean \pm s.d. ${ }^{*} P<0.05$.
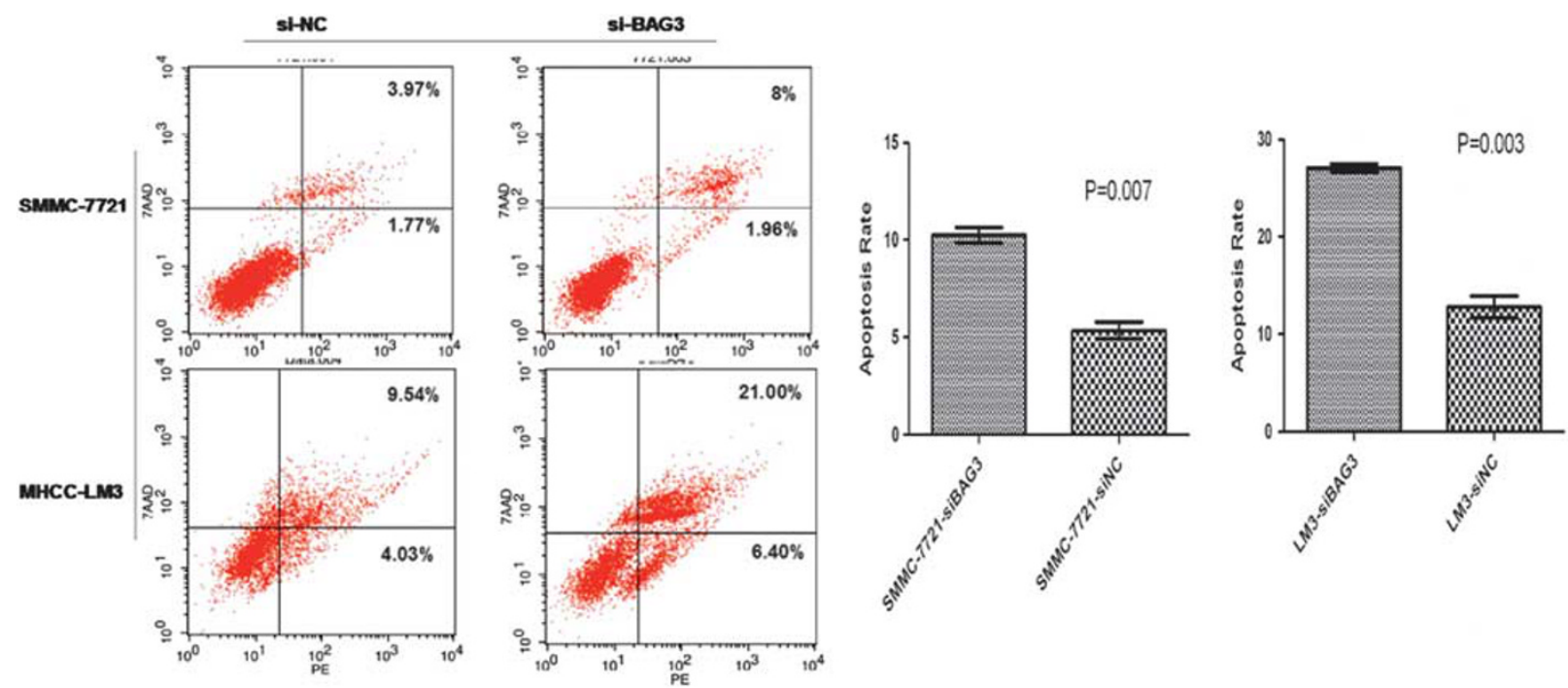

Figure 3 Knockdown of BAG3 induces apoptosis of HCC cell lines. A flow-cytometry method was adopted to analyze the effect of BAG3 RNAi on the frequency apoptosis of HCC cells by PE/7AAD staining. Data are expressed as mean \pm s.d.

control cells were injected subcutaneously into nude mice (five animals per group). All mice were killed and the tumors were dissected at end of the experiment. Compared with the controls, BAG3 knockdown resulted in a significant decrease in tumor size and weight $(P<0.001)$ (Figure 6b). The tumors were stained with CD34, VEGF, MMP-2, E-cadherin, and vimentin. The immunoreactivity and expression of CD34, VEGF, MMP-2, and vimentin were decreased in BAG3 knockdown-injected tumors as compared with negative control mice, and the expression of E-cadherin was increased. Consistent with immunohistochemistry results, western blot analysis showed the same phenomenon (Figures $6 \mathrm{c}$ and $\mathrm{d}$ ).

\section{HIF-1a and ERK Phosphorylation Are Essential for BAG3-Mediated VEGF and MMP-2 Expression and BAG3 can Regulate Autophagy through LC3A/B and Atg5 Expression}

HIF-1a has a significant role in regulating angiogenesis under hypoxic conditions, through regulation of target genes including VEGF and MMPs. ${ }^{16,17}$ Thus, we analyzed HIF-1a expression by western blotting. We found that HIF-1a was 

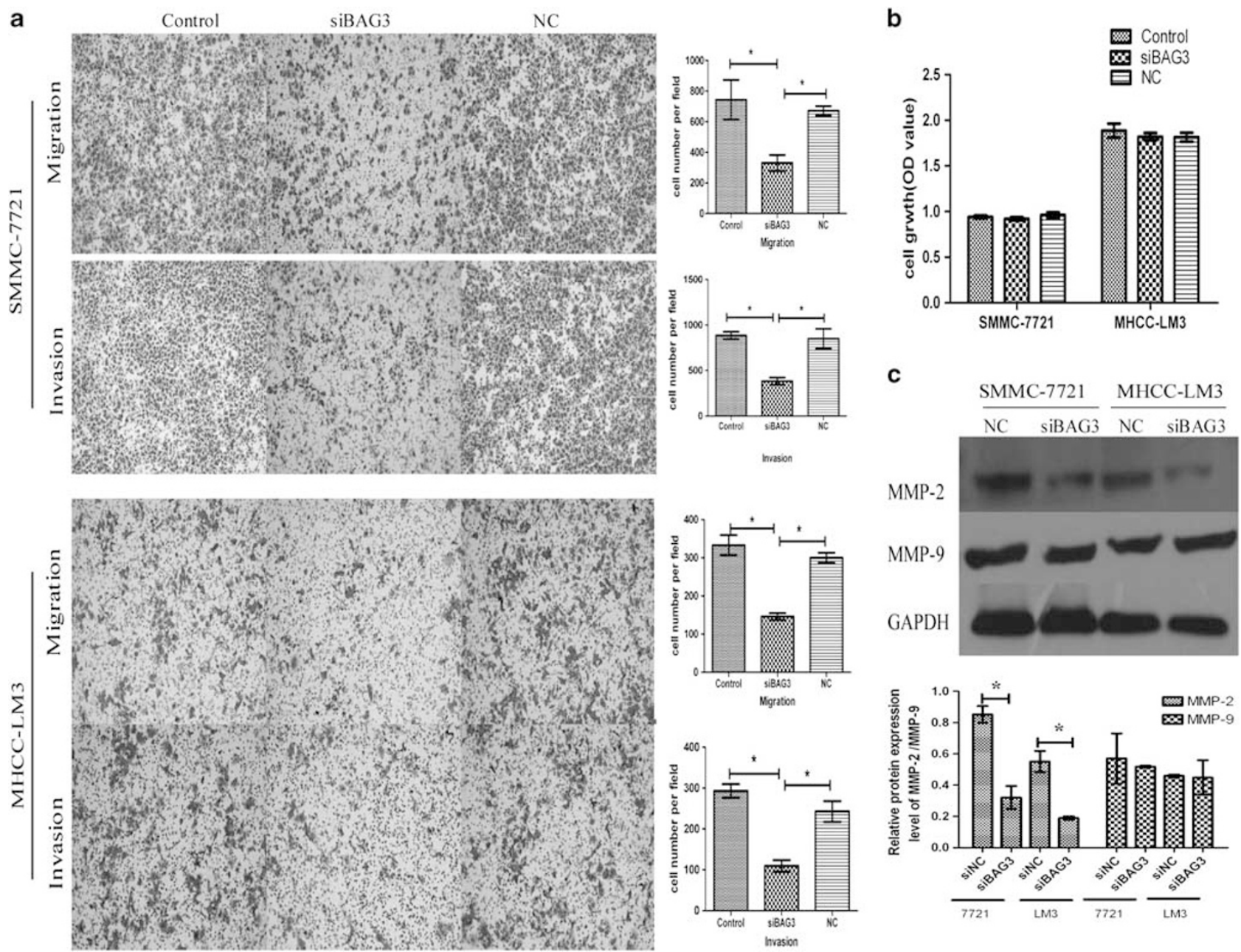

Figure 4 Knockdown of BAG3 inhibits migration and invasion of HCC cell lines. (a) Migration and invasion of stable BAG3 knockdown cells was measured by a transwell assay. Results were quantitated by counting migrating and invasive cells in five randomly chosen high-power fields for each replicate $(n=3)$. (b) Cell proliferation was measured by a CCK-8 assay after cells were incubated for $48 \mathrm{~h}(n=8)$. (c) Effects of BAG3 knockdown on MMP-2 and MMP-9 were measured by western blotting. GAPDH was used as a control. Experiments were performed three times. Data are expressed as mean \pm s.d. ${ }^{*} P<0.05$.

significantly decreased in MHCC-LM3 cell lines (Figure 7). Furthermore, it has been reported that BAG3 controls angiogenesis of endothelial cells and tumor cells through the ERK phosphorylation, ${ }^{12}$ so we examined the expression levels of ERK and p-ERK. We found that ERK phosphorylation was higher after BAG3 knockdown than the control group (Figure 7). Recent reports point to a role for BAG3 in controlling autophagy-related genes which could, in turn, regulate angiogenensis. ${ }^{18,19}$ We analyzed autophagy markers (LC3A/B and Atg5) by western blotting. We found that Atg5 was significantly decreased after BAG3 knockdown, and that LC3A/B was decreased in the MHCCLM3 cell line (Figure 7).

\section{DISCUSSION}

BAG3 has been studied in many primary tumors and tumor cell lines. ${ }^{4-10}$ However, there are few reports on the relationship between BAG3 function and HCC. The findings of this study are, first, that tumor tissue and cell line studies demonstrate that BAG3 is highly expressed in tumor tissues, compared with the adjacent tissues, as well as in HCC cell lines. Second, BAG3 regulates the invasion and EMT of HCC cell lines through regulating MMP-2, E-cadherin, slug, and vimentin expression. Third, the possible molecular mechanisms of BAG3 in angiogenesis are through the HIF1a signaling pathway and ERK phosphorylation. Fourth, BAG3 expression directly regulates the tumorigenicity of HCC cell lines in vivo. All in all, these results provide the first evidence supporting the pro-oncogenic and pro-metastatic function of BAG3 in HCC.

The potential of BAG3-targeting therapy for HCC has not been explored. Here, we showed that BAG3 knockdown effectively suppressed the tumor cell migration and invasion in vitro. A possible mechanism of role of BAG3 in the metastasis and invasion of HCC cells is MMP-mediated ECM degradation. ${ }^{20-22}$ Recently, some studies have reported that 
a
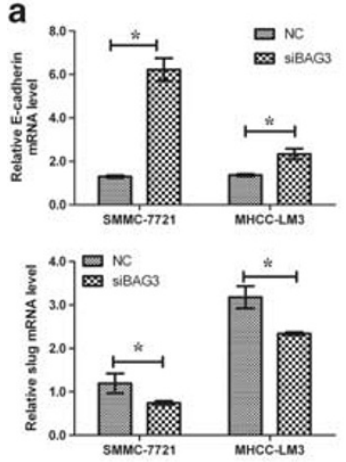
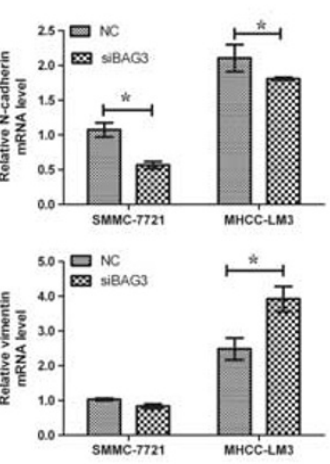

b
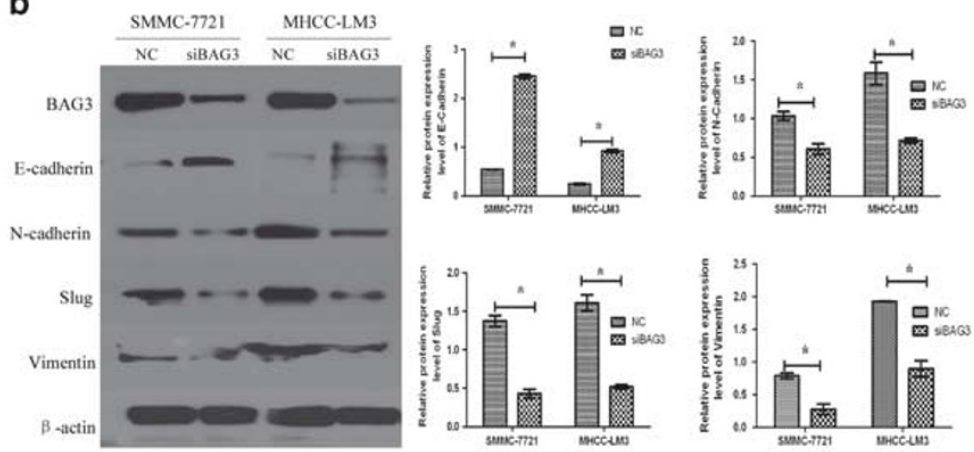

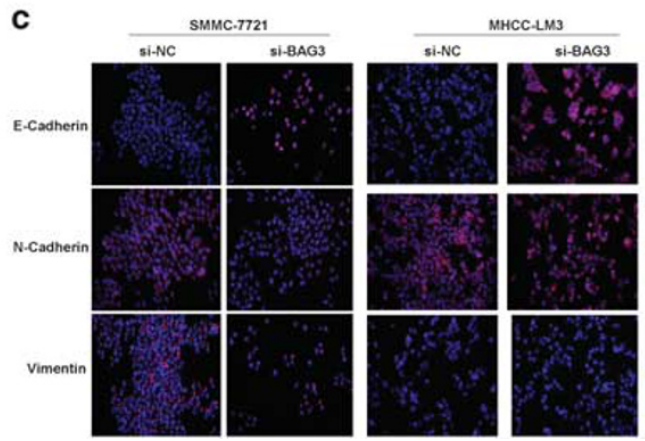

Figure 5 BAG3 knockdown reduces the EMT of HCC cell lines. (a) The mRNA levels of EMT marker genes such as E-cadherin, N-cadherin, slug, and vimentin were measured by real-time PCR. (b) The EMT marker (E-cadherin, N-cadherin, slug, and vimentin) protein levels were measured by western blotting. $\beta$-Actin was used as a control. (c) The EMT markers (E-cadherin, $\mathrm{N}$-cadherin, and vimentin) were measured by immunofluorescence. Data are expressed as mean \pm s.d. $* P<0.05$.

BAG3 may promote the motility and adhesion of epithelial cancer cells and interact with MMP-2 to regulate invasion of ovarian carcinoma cells. ${ }^{9,23}$ In the current study, we demonstrated that BAG3 knockdown resulted in a marked reduction in protein levels of MMP-2 in HCC cells in vitro, but the protein level of MMP-9 was not changed. Although some reports have demonstrated a critical role of MMP-9 in invasion, and slug might interact with MMP-9 expression to regulate invasiveness of tumor cells, ${ }^{24,25}$ MMP-9 expression did not correlate with slug reduction in our study.

Another possible mechanism for BAG3 invasion in HCC is that EMT is involved in tumor progression. Downregulation of epithelial gene expression such as E-cadherin results in loss of cell adhesion and polarity, while upregulation of mesenchymal gene expression ( $\mathrm{N}$-cadherin, vimentin, and slug) enhances cell motility and invasion. ${ }^{26,30}$ Our study demonstrates that BAG3 knockdown reverses EMT by increasing E-cadherin expression and decreasing $\mathrm{N}$-cadherin, vimentin, and slug expression in vitro and in vivo. It has been reported that slug is one of the transcriptional suppressors of E-cadherin, and downregulation of slug results in upregulation of E-cadherin. ${ }^{25-32}$ Slug is one of the potential predictive biomarkers for identifying patients with lymph-node metastasis in colorectal cancer. ${ }^{33}$ Moreover, BAG3 expression levels were significantly higher in HCC with portal vein-emboli and metastasis in our clinical analysis, and upregulation of slug in tissue in conjunction with an increase in BAG3 could reflect that cross-talk between BAG3 and EMT occurs in human HCC. Combined with no evident correlation between $\mathrm{E}$-cadherin, $\mathrm{N}$-cadherin, or vimentin with BAG3 in clinical HCC samples, it is likely that BAG3-induced metastasis is independent of slug expression.

In our study, BAG3 knockdown effectively suppressed the tumorigenicity of HCC cell lines in vivo. This finding is in accordance with previous studies in glioblastoma and thyroid carcinoma. ${ }^{10,34}$ A potential mechanism is that BAG3 knockdown may decrease the tumor neovascularization that supplies the tumor cells with nutrients, ${ }^{12}$ thereby inhibiting the tumorigenicity and metastasis of HCC cell lines in vivo. In the current study, we measured CD34 and VEGF expression in vivo. We found that CD34 and VEGF were decreased in BAG3 knockdown-injected tumors. In this study, we observed a role for HIF-1a in BAG3-regulated VEGF and MMP2 expression which was consistent with previous reports that HIF-1a promotes VEGF and MMP2 expression, enhancing angiogenesis and metastasis of HCC. ${ }^{16-35}$ Moreover, BAG3 regulated angiogenesis of HCC cells through ERK phosphorylation, in accordance with previous studies. ${ }^{12}$ Earlier work showed that autophagy-related genes could downregulate angiogenesis; ${ }^{18,19}$ however, in our study, knockdown of BAG3 could downregulate the autophagy process. According to these data, a possible molecular 
a

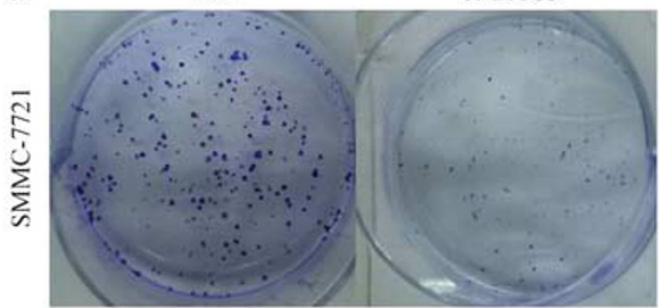

$\mathrm{Si}-\mathrm{BAG} 3$

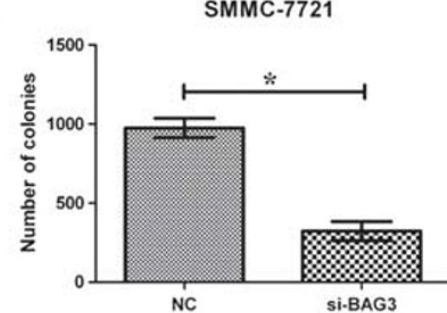

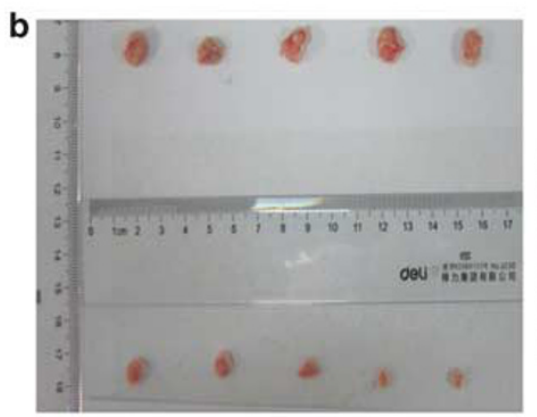

C

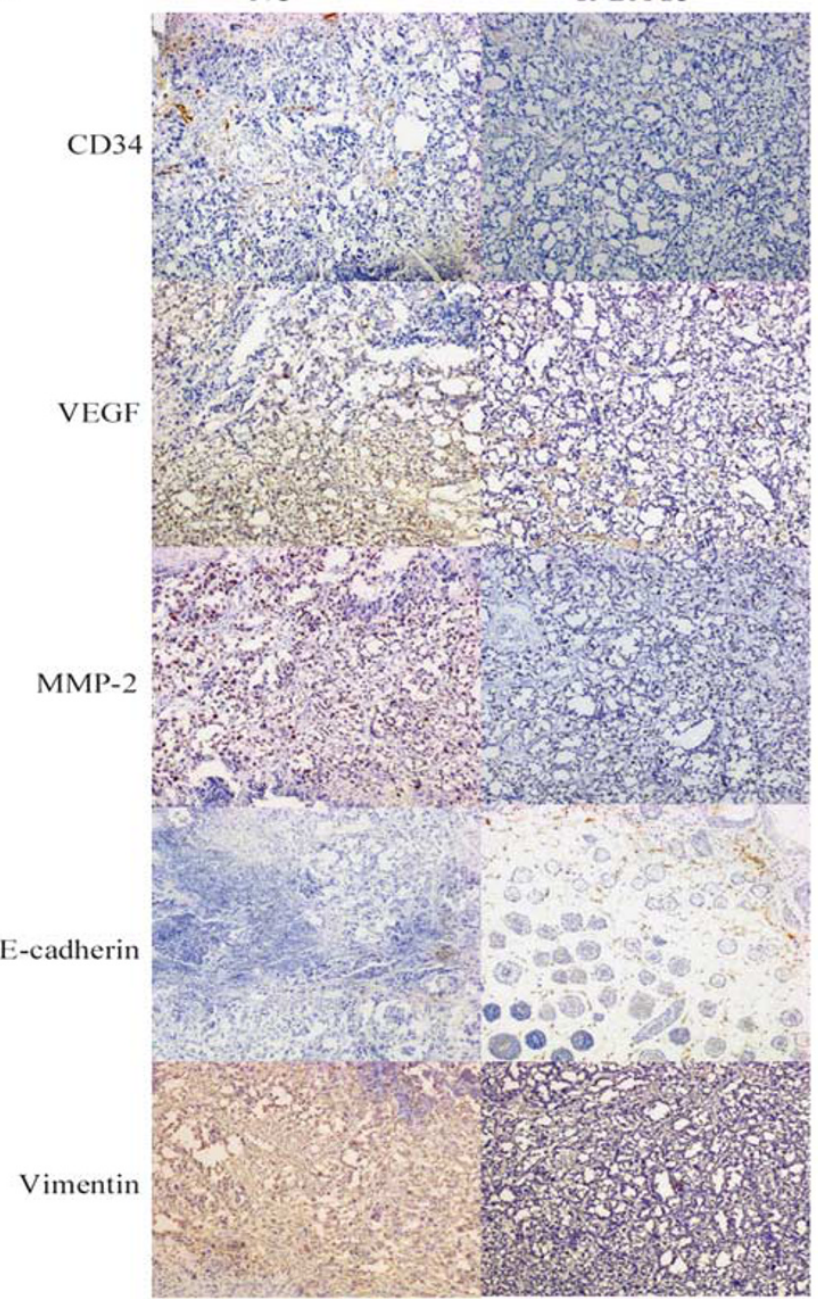

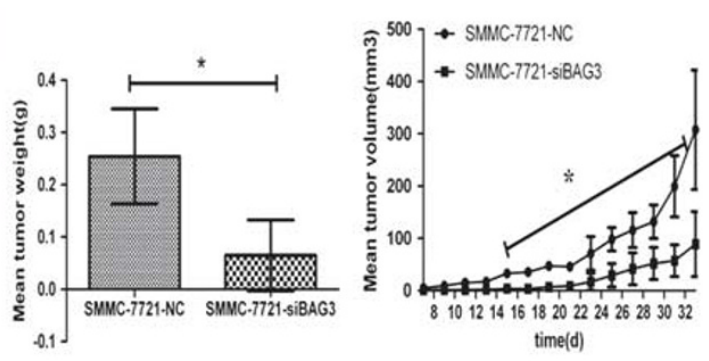

d
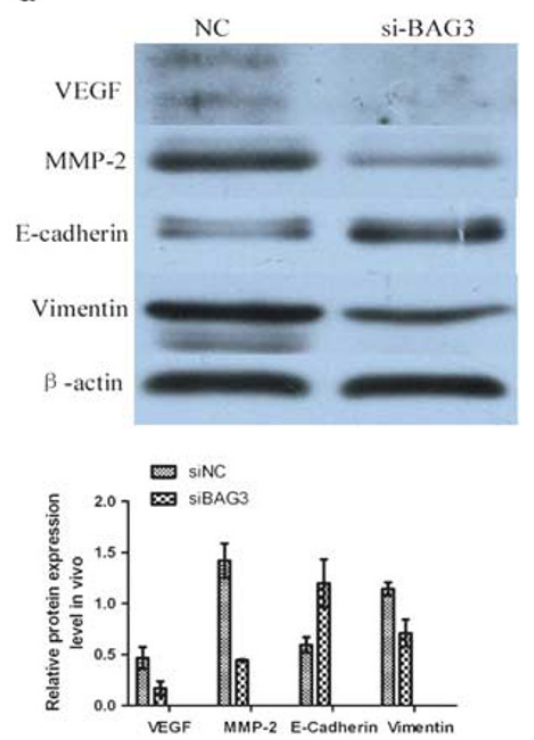

Figure 6 BAG3 knockdown inhibits the tumorigenicity of HCC cells. (a) Colony forming assay: BAG3 knockdown inhibits HCC cells colony formation. (b) In vivo tumorigenesis assay of tumor size and weight after transfected HCC cells were injected subcutaneously into nude mice. (c, d) Immunohistochemistry and western blot analysis of CD34, VEGF, MMP-2, E-cadherin, and vimentin. $\beta$-Actin was used as a control. Data were expressed as mean \pm s.d. ${ }^{*} P<0.05$. 

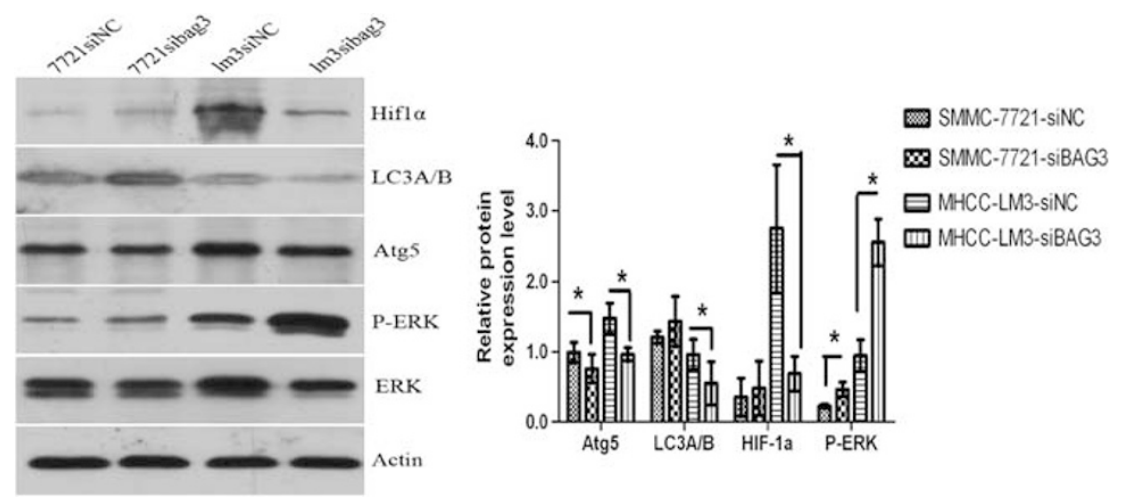

Figure 7 HIF-1a and ERK phosphorylation are essential for BAG3-mediated VEGF and MMP-2 expression and BAG3 can regulate autophagy through LC3A/B and Atg 5 expression. The protein levels of HIF-1a, ERK, $\mathrm{p}$-ERK, LC3A/B, and Atg 5 were measured by western blotting. $\beta$-Actin was used as a control. Data are expressed as mean \pm s.d. ${ }^{*} P<0.05$.

mechanism of BAG3 in the metastasis and angiogenesis of HCC cells involves the HIF-1a signaling pathway and ERK phosphorylation.

In conclusion, we demonstrated that BAG3 knockdown is associated with the invasiveness of HCC cells, potentially by inhibiting EMT and angiogenesis. BAG3 knockdown also effectively suppressed the tumorigenicity of HCC cell lines. We suggest that control of invasion and metastasis through inhibition of BAG3 gene may contribute to a novel therapeutic approach against HCC.

\section{ACKNOWLEDGMENTS}

This study was sponsored by grants from National High Technology Research and Development Program 863 of China (No. 2012AA021002), Special Fund for Health Research in the Public Welfare (201302009), and Zhejiang Provincial Natural Science Foundation for Young Distinguished Scholars (No. R2110125).

\section{DISCLOSURE/CONFLICT OF INTEREST}

The authors declare no conflict of interest.

1. Takayama S, Reed JC. Molecular chaperone targeting and regulation by BAG family proteins. Nat Cell Biol 2001;3:237-241.

2. Takayama S, Xie Z, Reed. JC. An evolutionarily conserved family of Hsp70/Hsc70 molecular chaperone regulators. J Biol Chem 1999;274: 781-786.

3. Rosati A, Ammirante M, Gentilella A, et al. Apoptosis inhibition in cancer cells: a novel molecular pathway that involves BAG3 protein. Int J Biochem Cell Biol 2007;39:1337-1342.

4. Romano MF, Festa M, Petrella A, et al. BAG3 protein regulates cell survival in childhood acute lymphoblastic leukemia cells. Cancer Biol Ther 2003;2:508-510.

5. Chiappetta G, Ammirante M, Basile A, et al. The antiapoptotic protein BAG3 is expressed in thyroid carcinomas and modulates apoptosis mediated by tumor necrosis factor-related apoptosis-inducing ligand. J Clin Endocrinol Metab 2007;92:1159-1163.

6. Gentilella A, Passiatore G, Deshmane $\mathrm{S}$, et al. Activation of BAG3 by Egr-1 in response to FGF-2 in neuroblastoma cells. Oncogene 2008; 27:5011-5018.

7. Staibano $S$, Mascolo $M$, Benedetto $M$, et al. BAG3 protein delocalisation in prostate carcinoma. Tumor Biol. 2010;31:461-469.

8. Wang H-Q, Meng X, Gao Y-Y, et al. Characterization of BAG3 cleavage during apoptosis of pancreatic cancer cells. J Cell Physiol 2010;224: 94-100.
9. Suzuki $M$, Iwasaki $M$, Sugio $A$, et al. BAG3 (BCL2-associated athanogene 3 ) interacts with MMP-2 to positively regulate invasion by ovarian carcinoma cells. Cancer Lett 2011;303; 65-71.

10. Festa $M$, Del Valle $L$, Khalili $K$, et al. BAG3 protein is overexpressed in human glioblastoma and is a potential target for therapy. Am J Pathol 2011;178:2504-2512.

11. Rosati A, Graziano V, De Laurenzi V, et al. BAG3: a multifaceted protein that regulates major cell pathways. Cell Death Dis 2011;7:141.

12. Falco A, Festa $M$, Basile $A$, et al. BAG3 controls angiogenesis through regulation of ERK phosphorylation. Oncogene 2012;31:5153-5161.

13. Li N, Du ZX, Zong ZH, et al. PKC[delta]-mediated phosphorylation of BAG3 at Ser187 site induces epithelial-mesenchymal transition and enhances invasiveness in thyroid cancer FRO cells. Oncogene 2012;32: 4539-4548.

14. He J, Gu D, Wu X, et al. Major causes of death among men and women in China. N Engl J Med 2005;353:1124-1134.

15. Pang RC, Joh J, Johnson P, et al. Biology of hepatocellular carcinoma. Ann Surg Oncol 2008;15:962-971.

16. Semenza GL. HIF-1 and tumor progression: pathophysiology and therapeutics. Trends Mol Med 2002;8:S62-S67.

17. Déry M.-A.C., Michaud MD, Richard DE. Hypoxia-inducible factor 1: regulation by hypoxic and non-hypoxic activators. Int J Biochem Cell Biol 2005;37:535-540.

18. De A, De A, Papasian C, et al. Extract induces autophagy and inhibits human ovarian cancer cell proliferation, angiogenesis, growth of mouse xenograft tumors. PLoS ONE 2013;8:e72748.

19. Kim KW, Paul P, Qiao J, et al. Enhanced autophagy blocks angiogenesis via degradation of gastrin-releasing peptide in neuroblastoma cells. Autophagy 20139.

20. Stetler-Stevenson WG, Aznavoorian S, Liotta LA. Tumor cell interactions with the extracellular matrix during invasion and metastasis. Annu Rev Cell Biol 1993;9:541-573.

21. Sengupta $N$, MacDonald $\Pi$. The role of matrix metalloproteinases in stromal/epithelial interactions in the gut. Physiology 2007;22: 401-409.

22. Polette $M$, Nawrocki-Raby $B$, Gilles $C$, et al. Tumour invasion and matrix metalloproteinases. Crit Rev Oncol Hematol 2004;49:179-186.

23. Iwasaki M, Homma S, Hishiya $A$, et al. BAG3 regulates motility and adhesion of epithelial cancer cells. Cancer Res 2007;67:10252-10259.

24. Naber HPH, Wiercinska E, ten Dijke $P$, et al. Spheroid assay to measure TGF- $\beta$-induced invasion. J Vis Exp 2011;16:3337.

25. Zhang $\mathrm{K}$, Chen $\mathrm{D}$, Jiao $\mathrm{X}$, et al. Slug enhances invasion ability of pancreatic cancer cells through upregulation of matrix metalloproteinase-9 and actin cytoskeleton remodeling. Lab Invest 2011;91: 426-438.

26. Huber MA, Kraut N, Beug H. Molecular requirements for epithelialmesenchymal transition during tumor progression. Curr Opin Cell Biol 2005; 17:548-558.

27. Mimeault M, Batra SK. Interplay of distinct growth factors during epithelial-mesenchymal transition of cancer progenitor cells and 
molecular targeting as novel cancer therapies. Ann Oncol 2007:18: 1605-1619.

28. Andersen $\mathrm{H}$, Mejlvang $\mathrm{J}$, Mahmood $\mathrm{S}$, et al. Immediate and delayed effects of E-cadherin inhibition on gene regulation and cell motility in human epidermoid carcinoma cells. Mol Cell Biol 2005;25: 9138-9150.

29. Thiery JP, Acloque $\mathrm{H}$, Huang RYJ, et al. Epithelial-mesenchymal transitions in development and disease. Cell 2009;139:871-890.

30. Arias AM. Epithelial mesenchymal interactions in cancer and development. Cell 2001;105:425-431.

31. Bolós V, Peinado H, Pérez-Moreno MA, et al. The transcription factor Slug represses E-cadherin expression and induces epithelial to mesenchymal transitions: a comparison with Snail and E47 repressors. J Cell Sci 2003:116:499-511.
32. Naber HPH, Drabsch Y, Snaar-Jagalska BE, et al. Snail and Slug, key regulators of TGF- $\beta$-induced EMT, are sufficient for the induction of single-cell invasion. Biochem Biophys Res Commun 2013;435:58-63.

33. Toiyama $Y$, Yasuda H, Saigusa S, et al. Increased expression of Slug and Vimentin as novel predictive biomarkers for lymph node metastasis and poor prognosis in colorectal cancer. Carcinogenesis 2013;34: 2548-2557.

34. Ammirante $\mathrm{M}$, Rosati $\mathrm{A}$, Arra $\mathrm{C}$, et al. IKK $\gamma$ protein is a target of BAG3 regulatory activity in human tumor growth. Proc Natl Acad Sci 2010; 107:7497-7502.

35. Fu J, Chen Y, Cao J, et al. p28GANK overexpression accelerates hepatocellular carcinoma invasiveness and metastasis via phosphoinositol 3-kinase/AKT/hypoxia-inducible factor- $1 \alpha$ pathways. Hepatology 2011:53:181-192. 